\title{
Aplicación clínica de la radioterapia de intensidad modulada
}

\section{The clinical application of intensity-modulated radiation therapy}

\author{
A. Manterola, P. Romero, G. Asín, M. Rico, A. Sola, M.T. Vila
}

\section{RESUMEN}

La radioterapia de intensidad modulada (IMRT) representa una de las mayores innovaciones técnicas de la moderna radioterapia. Su capacidad de conseguir tratamientos con la dosis altamente conformada al área de irradiación permite tratar volúmenes próximos a órganos de riesgo con gran seguridad. Estas características la convierten en una técnica ideal para estudios, bien de disminución de toxicidad en órganos de riesgo, bien de intensificación de dosis para mejorar el control de la enfermedad. La primera parte de este artículo tratará sobre qué se entiende por IMRT y sus peculiares características dosimétricas, así como de los tipos de IMRT; en la segunda parte se tratará la evidencia clínica en algunas de las localizaciones más investigadas como son tumores de cabeza y cuello, próstata y mama.

Palabras clave. IMRT. Radioterapia. Cáncer.

\begin{abstract}
Intensity-modulated radiation therapy (IMRT) represents one of the greatest technical innovations in modern radiotherapy. Its capacity of achieving treatments with the dose conforming largely to the irradiated area makes it possible to treat volumes close to organs at risk with great safety These characteristics make it an ideal technique for studies, whether for reducing toxicity in organs at risk, or for intensifying dosages to improve the control of the disease. The first part of the article considers what is understood by IMRT and its peculiar dosimetric characteristics, as well the types of IMRT; the second part deals with the clinical evidence in some localisations such as tumours of the head and neck, prostate and breast.
\end{abstract}

Key words. IMRT. Radiotherapy. Cancer
Servicio de Oncología Radioterápica. Hospital de Navarra. Pamplona.

\section{Correspondencia}

Ana Manterola Burgaleta

Servicio de Oncología Radioterápica

Hospital de Navarra

Irunlarrea, 3

31008 Pamplona

Tfno. 848428475

E-mail: ana.manterola.burgaleta@cfnavarra.es 


\section{INTRODUCCIÓN}

Desde su introducción en la práctica clínica a finales de los años 90, la radioterapia de intensidad modulada (IMRT) ha generado un gran interés y representa una de las mayores innovaciones técnicas en la moderna radioterapia (RT).

La IMRT es un tratamiento tridimensional conformado avanzado que es capaz de generar un haz de irradiación de intensidad no uniforme: asigna de forma óptima diferentes "pesos" a pequeñas subdivisiones de los haces llamados "rays" o "beamlets".

La posibilidad de manipular la intensidad de cada individual "ray" dentro de cada haz, permite un gran control sobre la fluencia de la radiación, consiguiendo una óptima distribución de dosis ${ }^{1}$.

La IMRT, combinada con técnicas de imagen guiada dirigida a unos volúmenes de tratamiento delineados con precisión, puede mejorar el control local y reducir la toxicidad en tejidos sanos.

\section{TEÓRICOS BENEFICIOS DE LA IMRT}

\section{Conformación de la dosis}

La mayor ventaja de la IMRT es su capacidad de producir una mayor conformación de la distribución de la dosis (es decir, que la dosis se adapta de una manera más precisa a la forma tridimensional del área que queremos tratar) que con los tratamientos 3-D convencionales (3DCRT). Característicamente, puede obtener distribuciones de dosis con concavidades localizadas entre, por un lado, el tumor u órgano a tratar (volumen blanco) y, por otro, el órgano a riesgo (OAR) que debe protegerse de las dosis altas de irradiación.

Por el contrario, las distribuciones de dosis obtenidas con 3DCRT son convexas o rectas y esto puede resultar subóptimo en algunas localizaciones como en el tratamiento de la próstata por su peculiar situación en relación con el recto, que es un órgano de riesgo muy sensible a la radiación y localizado muy próximo a la zona de altas dosis.

\section{Seguridad de los órganos no afectos}

A la habilidad para conseguir un tratamiento altamente conformado se une la de obtener alto gradiente de dosis (fuertes caídas de dosis alrededor del volumen blanco), esto significa que las dosis en los OAR próximos pueden reducirse mucho. Así, podemos obtener diferentes beneficios, bien disminuir la toxicidad por la menor dosis recibida en los OAR, o bien conseguir aumentar la dosis en el volumen blanco sin aumentar la toxicidad en tejidos adyacentes.

Por esto, la IMRT tiene el mayor impacto clínico en los tratamientos que requieren una gran conformación o un fuerte gradiente de dosis entre áreas a tratar y órganos de riesgo. Esto ocurre cuando ambos se encuentran muy próximos entre sí y sobre todo si el OAR empuja el contorno del área a tratar y penetra en una concavidad del mismo (ej. el recto en el tratamiento de cáncer prostático o la médula espinal en el tratamiento de tumores de cabeza y cuello) (Fig. 1).

\section{Homogeneidad de dosis}

Es posible un mayor control del gradiente de dosis en el área de tratamiento en sentidos opuestos; una de las posibilidades es la de obtener una alta homogeneidad de dosis en el órgano a tratar, por ejemplo en irradiación de mama evitando así zonas infradosificadas (riesgo de disminuir el control local) y áreas sobredosificadas (riesgo de mayor toxicidad). Por otro lado, existe la posibilidad de obtener diferentes niveles de dosis de forma simultánea. Los diferentes volúmenes blanco (área de enfermedad macroscópica, área de enfermedad subclínica o tratamientos ganglionares electivos) necesitan diferentes dosis; tradicionalmente esto se ha conseguido con irradiaciones secuenciales realizadas en diferentes fases del tratamiento y con planificaciones independientes. Con la IMRT, estas múltiples fases se pueden integrar en un único plan de tratamiento durante todo el curso 

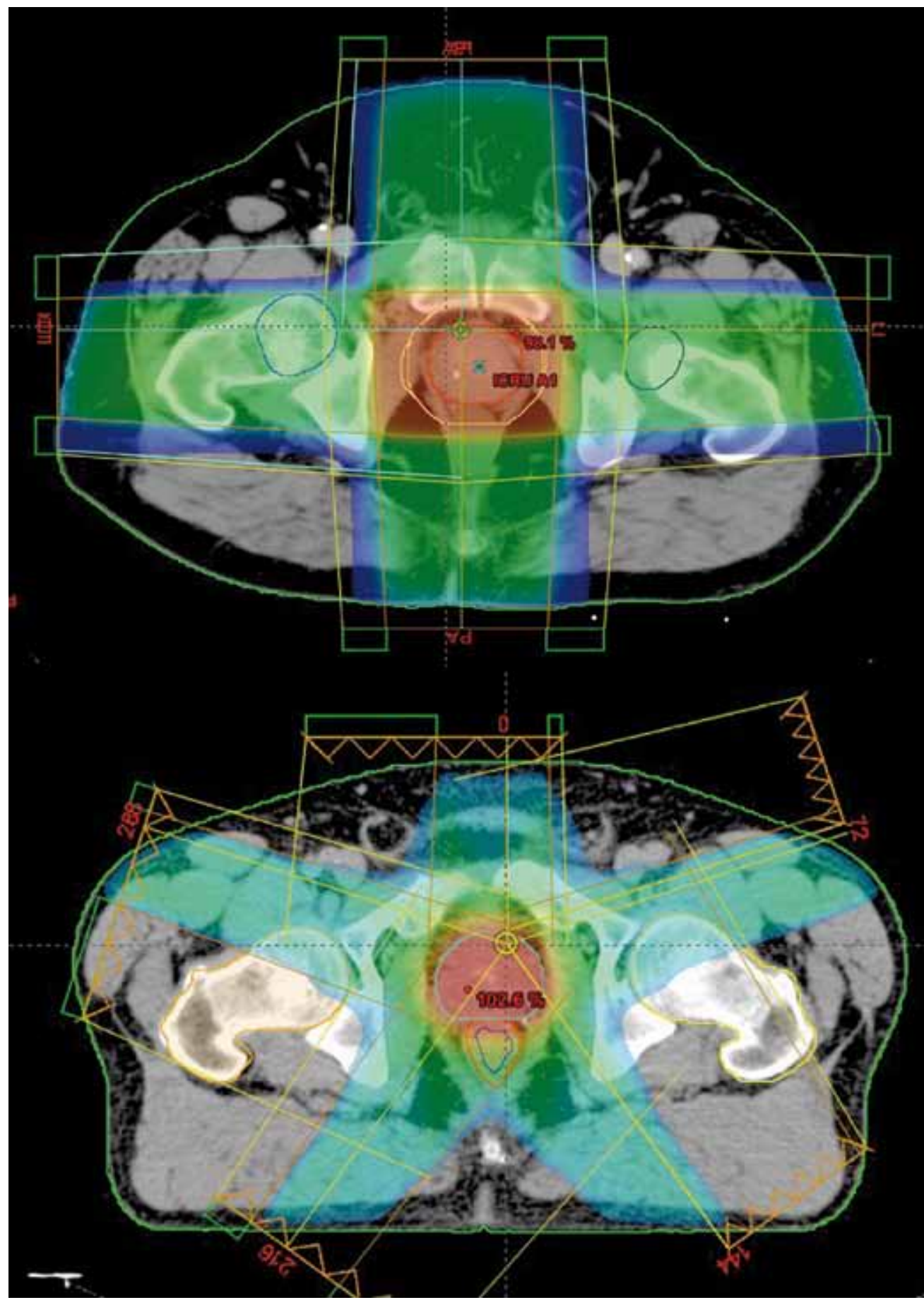

Figura 1. Superior: dosimetría de irradiación prostática con planificación convencional 3D. Inferior: planificación IMRT. El área de dosis terapéutica (área roja) se adapta mucho mejor al volumen blanco con IMRT. 
de dicho tratamiento (ej: tratamientos de boost -o sobredosificación- integrado simultáneo -SIB-) y así reducir la duración de la RT. También permite, por ejemplo, aumentar la dosis prescrita a la próstata para mejorar el control local, pero manteniendo dosis moderadas alrededor de la uretra.

Esta estrategia puede tener también ventajas radiobiológicas, ya que mientras un área determinada recibirá una dosis/fracción moderada, en el mismo tratamiento, otra zona estará recibiendo una dosis/fracción alta. Esto puede ser beneficioso si estamos tratando áreas de diferente radiosensibilidad en el mismo tumor (ej. las áreas hipóxicas presentan menor radiosensibilidad) y nos podemos beneficiar de los grandes avances en imagen funcional para optimizar nuestra planificación.

Es importante saber que estas tres ventajas o posibilidades de la IMRT suponen lo que Pirzkall llamó objetivos "en competencia" en el volumen blanco ${ }^{2}$ : la compleja geometría del tratamiento impide la consecución simultánea de las tres. Es decir que tenemos que dar una mayor o menor prioridad en nuestro plan de tratamiento a la homogeneidad de dosis en el volumen blanco según el caso. En general y debido a las ventajas que se pueden obtener en áreas con geometrías difíciles, se prefiere la mayor conformación con aumento en la prescripción de dosis, asumiendo en consecuencia un aumento en la heterogeneidad dosimétrica, y siempre sin olvidar que si permitimos áreas de sobredosificación podemos exponer al paciente a una severa toxicidad (ej. zonas de mucosa, nervios, óseas en tratamientos de cabeza y cuello) y por el contrario zonas de infradosificación (generalmente próximas a OAR) pueden suponer una pérdida del control tumoral, no compensada por las áreas de sobredosificación.

\section{Calidad del plan de tratamiento}

Los complicados cálculos que se precisan requieren el uso de optimización computarizada especializada; la óptima intensidad de cada "beamlet" (pequeña subdivisión del haz con una determinada intensidad de radiación) se realiza mediante un proceso sistemático reiterativo por el cual el ordenador genera planes de intensidad modulada secuenciales. Uno a uno, los va evaluando de acuerdo a un determinado criterio ("objetivos deseados") y realiza cambios en la intensidad de estos "beamlet", basándose en la desviación de los "objetivos deseados". A esto se denomina "planificación inversa", donde se definen en primer lugar unos objetivos y el programa de cálculo dosimétrico investiga cuál es la óptima modulación del haz para conseguirlos. Contrariamente, en la planificación utilizada previamente a la IMRT (planificación directa o hacia delante: "forward planning") primero se realizan los cálculos de dosis y después se valora si ese resultado se adapta con mayor o menor fidelidad a los objetivos y, si no es así, se modifican las condiciones de los haces y se vuelve a calcular. La calidad del plan de tratamiento depende de múltiples factores:

- Criterios de optimización utilizados para comparar planes alternativos.

- Los algoritmos de optimización y de cálculo de dosis.

- Características de los haces: número, orientación y energía.

- Márgenes asignados al PTV y/o tejidos normales.

Estas situaciones requieren un fuerte implicación del clínico en el proceso de planificación ya que es necesario establecer un compromiso entre los objetivos buscados que, en un momento dado, pueden interferir unos con otros (por ejemplo, si el objetivo principal es que toda la próstata reciba una dosis de 80 Gys, no se podrá disminuir la irradiación de ciertas zonas del recto por debajo de determinada dosis). También es cierto que la mayor conformación ha permitido prescribir dosis más altas, pero a menudo, como ya hemos visto, con un aumento de la heterogeneidad en el volumen blanco. 


\section{LIMITACIONES Y RIESGOS}

\section{Limitaciones}

- Muchas distribuciones no son físicamente posibles: se han expuesto las ventajas de la IMRT pero, es muy probable que no se puedan conseguir todos estos beneficios en una misma planificación. Se deberá priorizar en cada caso lo que más nos interese.

- Nuestro conocimiento sobre qué es óptimo clínicamente y cómo definir mejor los objetivos de la IMRT es limitado.

- Limitada experiencia en los cambios de dosis/fracción (ejem: SIB), diversas incertidumbres pueden limitar la aplicabilidad y la eficacia de la IMRT:

- El volumen blanco puede variar entre una fracción y la siguiente (variaciones interfracción) por posicionamiento o cambios anatómicos.

- Variaciones en la localización del volumen blanco durante el curso de la sesión de tratamiento (variaciones intrafracción), por ejemplo, por movimientos respiratorios.

- Cambios de las características físicas y radiobiológicas del tumor.

- Aumento de las unidades de monitor (el paciente debe permanecer más tiempo recibiendo la sesión de RT para alcanzar una dosis similar que sin IMRT) con el consiguiente aumento de la radiación dispersa y de la transmisión a través de los sistemas de conformación del haz (colimadores multiláminas: MLC).

- Limitación de los sistemas de verificación de la irradiación administrada.

- Falta de precisión de la tecnología de la imagen en la definición de la verdadera extensión del tumor y de las características radiobiológicas, así como las de los tejidos sanos.

\section{Riesgos}

- Posibilidad de pérdidas geométricas en la cobertura del volumen blanco con la utilización de márgenes muy ajustados y sobre todo en áreas de incertidumbre debida al movimiento, dificultades en el posicionamiento, etc.

- Debido a la posibilidad de obtener altos gradientes de dosis entre volumen blanco y tejidos sanos, existe mayor peligro de que un tejido sano reciba una alta dosis de RT.

- Obtención de distribuciones de dosis complejas y nuevas, difíciles de evaluar e interpretar su eficacia.

Por todos estos motivos se exige un control de calidad muy estricto donde tiene gran importancia disminuir la incertidumbre del posicionamiento diario y los movimientos del volumen blanco durante la RT. Si tenemos en cuenta que una de las características de la IMRT es la conformación de la dosis al volumen blanco, las desviaciones de dosis que podemos obtener respecto al plan teórico de irradiación son mucho mayores que con técnicas de 3-DRT y por lo tanto tendrán más repercusión en los resultados del tratamiento. Para evitar esto, se han desarrollado mecanismos especiales de inmovilización, de verificación del posicionamiento y otros complejos sistemas de irradiación como es el "gating" en el que la RT sólo se administra durante un determinado período del ciclo respiratorio.

\section{TIPOS DE RADIOTERAPIA DE INTENSIDAD MODERADA}

Durante los últimos 15 años se han diseñado distintas técnicas para optimizar la IMRT $^{1}$. Las diferencias más importantes se deben a los mecanismos que utilizan para administrar las fluencias no uniformes del haz de radiación (Tabla 1). De entre los distintos tipos existen 2 dominantes y claramente diferentes: tomoterapia e IMRT "convencional". 
Tabla 1. Tipos de IMRT.

MLC: Colimador multiláminas
IMAT: Intensidad modulada con arco-terapia

\section{Tomoterapia}

El tratamiento se administra mediante un haz muy fino que va rotando alrededor del paciente (como en una unidad de TAC). Mientras el haz gira, los colimadores multiláminas van entrando y saliendo del mismo y así se realiza la conformación del haz. De esta manera, en cada punto del giro, el haz tendrá una anchura determinada y diferente según las necesidades dosimétricas.

De forma similar al funcionamiento de un TAC, la mesa donde se apoya el paciente se desplaza hasta cubrir toda la longitud del volumen a irradiar, bien realizando un desplazamiento discreto entre sucesivas rotaciones (tomoterapia seriada) o bien mediante un movimiento continuo a la vez que gira el haz (tomoterapia helicoidal).

En la modalidad helicoidal, el sistema posee un TAC convencional que incluye un portal detector de megavoltaje para realizar reconstrucciones tomográficas de la distribución de dosis.

\section{IMRT "convencional"}

A diferencia de la tomoterapia, la apertura del haz puede ser lo suficientemente grande para cubrir toda el área de tratamiento, de forma que un tratamiento podría administrarse durante un único giro del haz. A su vez existen 2 subgrupos:
1. Brazo fijo: la irradiación se administra con el brazo del acelerador lineal en una posición fija y una vez finaliza, el brazo se coloca en la siguiente posición y vuelve a irradiar. Existen dos modalidades según el funcionamiento de los MLC: modo dinámico ("sliding window") en la que los colimadores se mueven durante la irradiación. La segunda modalidad es el modo estático ("step and shot") en el que la fuente no emite radiación hasta que los colimadores han alcanzado su posición y están parados.

2. IMRT "arc therapy" (IMAT): el brazo va girando durante el tratamiento $\mathrm{y}$ los MLC se van moviendo al mismo tiempo.

\section{EXPERIENCIA CLÍNICA CON IMRT}

\section{Cabeza y cuello}

Dos estrategias se pueden diferenciar en la investigación de la IMRT en tumores de cabeza y cuello. La primera, manteniendo la dosis por fracción convencional (200 cGys) en las áreas de enfermedad clínica y administrando menor dosis/fracción en áreas de enfermedad subclínica con la intención de disminuir la toxicidad en OAR (parótidas, glándulas lacrimales, vías ópticas, oído medio o lóbulo temporal entre 
otras) sin disminuir el control de la enfermedad.

En este sentido, uno de los primeros trabajos publicados que data de 1999, consiguió disminuir la dosis en las parótidas a menos de 30 Gys en 28 pacientes con tomoterapia seriada. Los autores describieron, así mismo una disminución de la toxicidad aguda $^{3}$. Un estudio prospectivo holandés recientemente publicado compara xerostomía aguda y tardía y calidad de vida (escalas RTOG) en 241 pacientes con tumores de cabeza y cuello tratados con RT (con/ sin QT) convencional 3-D y IMRT. La IMRT consiguió mejoría en los tres parámetros ${ }^{4}$.

En carcinoma de orofaringe, un estudio prospectivo de $\mathrm{Chao}^{5}$ sobre 430 pacientes demostró que la ventaja dosimétrica de la IMRT se traducía en una disminución de la toxicidad salivar tardía sin mermar la eficacia del tratamiento. Recientemente se han publicado los resultados preliminares del ensayo Fase II de la RTOG, H- $0022^{6}$ con buenos resultados en toxicidad aguda y crónica (seguimiento medio de 13,3 m).

En ciertas localizaciones como aquellas próximas a base de cráneo (nasofaringe y tumores sinonasales) la IMRT puede tener un claro beneficio terapéutico.

En tumores de nasofaringe se pudo demostrar una mejor cobertura de los ganglios con disminución de dosis en las parótidas, sin disminuir la cobertura del tumor primario ${ }^{7,8}$. Estas ventajas en la distribución de dosis se han traducido en excelentes resultados clínicos en relación a xerostomía ${ }^{9}$, calidad de vida ${ }^{10}$ y supervivencia libre de progresión ${ }^{11}$.

Los tumores de senos paranasales presentan una complicada situación entre importantes OAR muy sensibles a la radiación como son vías ópticas, estructuras orbitarias, hipófisis y tronco del encéfalo entre otras. Claus ${ }^{12}$ trató 11 pacientes con carcinoma de seno etmoidal sin producir "ojo seco" ni otras toxicidades oculares y salvando la visión binocular. Dosis máximas de 50 Gys en retina y 60 Gys en nervios ópticos y quiasma con fraccionamientos de 1,5-1,7 Gys se encuentran en el umbral de dosis-máxima tolerada para la preservación visual ${ }^{13}$.

La segunda estrategia propone utilizar una dosis mayor de 200 cGy por fracción (generalmente entre 220-240 cGy) al tumor. En este caso el objetivo es acelerar el tratamiento para conseguir mejor control tumoral, sin aumentar la toxicidad ${ }^{14}$.

Se han realizado estudios con escalada de dosis en carcinomas de cabeza y cuello avanzados con técnica de SIB ("simultaneus integrated boost") con dosis de 64,170,8-73,8 Gys (biológicamente equivalentes si utilizáramos un fraccionamiento de 200 cGys por fracción a 74, 79 y 85 Gys) sin sobrepasar las dosis tolerables en médula espinal y tronco del encéfalo ${ }^{15}$.

En tumores de laringe e hipofaringe, los datos clínicos con tratamientos IMRT son escasos. Un estudio reciente analiza la posibilidad de escalada de dosis incluyendo quimioterapia concomitante resultando una buena adherencia al tratamiento y aceptable toxicidad aguda ${ }^{16}$.

Una de los peligros al utilizar IMRT debido a la alta conformación y gradiente de dosis es la de que se produzcan "fallos marginales", es decir recidivas en las áreas adyacentes a la región de altas dosis. Varios trabajos han demostrado que las recidivas locales en tratamientos con IMRT se producen fundamentalmente en las áreas de alta dosis ${ }^{17,18}$. En este sentido, las nuevas técnicas de imagen como el PET y RM con espectroscopía tienen la capacidad de construir mapas tridimensionales con parámetros radiobiológicamente relevantes como son la hipoxia o índices proliferativos que puedan guiar la planificación de tratamientos con intensificación de dosis en estas áreas ${ }^{19}$. Este es el fundamento de la llamada IMRT-“dose-painting” en la que es posible adaptar la dosis administrada (dosis por fracción y dosis total) a la particular radiosensibilidad/radiorresistencia de las diferentes áreas tumorales.

\section{Próstata}

Diversos estudios randomizados y no randomizados han demostrado el benefi- 
cio de aumentar la dosis de RT para mejorar el control tumoral (aumento del 15$20 \%$ en control bioquímico) en cáncer de próstata. Con las técnicas convencionales no es posible pasar de ciertas dosis (7076 Gys) sin exceder la tolerancia de los OAR: vejiga, intestino y recto. Los estudios iniciales con IMRT demostraron una mejor cobertura del volumen blanco y una menor área de recto y vejiga que recibía dosis de 75 Gys frente a técnicas de 3DRT. Clínicamente esto se tradujo en una menor toxicidad aguda gastrointestinal con una tendencia a disminuir la toxicidad genitourinaria; además el riesgo de sangrado rectal G2-3 bajó del 10\% con 3D-RT al $2 \%$ con IMRT $^{20}$. En 2002, investigadores del MSKCC publicaron la posibilidad de administrar dosis de, al menos, 81 Gys en próstata en un estudio con 772 pacientes con excelentes datos de toxicidad y control de enfermedad ${ }^{21}$. Recientemente se ha publicado una actualización de estos datos con mediana de seguimiento de 7 años que corroboran los datos de seguridad del tratamiento y excelentes cifras de control bioquímico $^{22}$. Se conoce la existencia de una dosis-respuesta en cáncer de próstata, pero la dosis óptima necesaria para optimizar el control local está por determinar. Estudios del Fox Chase Cancer Center sugieren que algunos pacientes se pueden beneficiar de dosis por encima de 80 Gys y que puede existir un "plateau" en la curva de dosis-respuesta por encima de esa cifra $^{23}$. Recientemente se han publicado tratamientos con dosis de 86,4 Gys bien toleradas ${ }^{24}$.

Estudios comparativos con dosis de IMRT de 76 Gys frente a 3D-RT con 68,4-70 Gys demuestran similar tolerancia e incluso disminución de la toxicidad con IMRT, gracias a la mayor conformación de la dosis y disminución del volumen de recto y vejiga que recibe dosis mayores de 70 Gys.

Aprovechando las ventajas dosimétricas de la IMRT se están investigando tratamientos con hipofraccionamiento, en los que dosis de 70 Gys en fracciones de 2,5 Gys (equivalente en tumor a 79-81 Gys) se administran en un corto período de tiem- po ${ }^{25}$. Con un seguimiento de 45 meses las toxicidades tardías son mínimas (menor del 5 y $6 \%$ de toxicidad G2 o mayor GI o GU respectivamente) y las cifras de control bioquímico esperanzadoras.

Es importante conocer que las mejoras en toxicidad conseguidas se han traducido en una mejora en datos de calidad de vida. Dos estudios han demostrado un aumento en los parámetros de calidad de vida evaluados, comparando tratamientos de IMRT a 74-80 Gys frente 3D-RT y tratamientos convencionales hasta 66-72 Gys $^{26,27}$.

Uno de los principales problemas a la hora de administrar un tratamiento IMRT en próstata, que como sabemos, implica una muy alta conformación de las dosis al volumen target, con un fuerte gradiente de dosis alrededor para no sobredosificar OAR vecinos, es la dificultad de localizar diariamente la situación de la próstata con los controles habituales de radioterapia. La próstata es un órgano que varía su posición de un día para otro en función del llenado de vejiga y recto y además el diario posicionamiento del paciente implica ciertas incertidumbres. Es imprescindible por tanto, disponer de métodos específicos para su correcta localización antes de la realización de cada sesión de tratamiento si vamos a utilizar IMRT. En la actualidad se utilizan distintos sistemas como la realización diaria antes de la sesión de RT de Ecografías, TAC en la misma unidad de tratamiento o el implante en glándula prostática de marcadores metálicos (marcadores fiduciales) que sean visibles en los sistemas de verificación convencionales, para ajustar así, día a día, el tratamiento a los desplazamientos que la próstata haya podido sufrir.

\section{Mama}

Los estudios con IMRT en mama van dirigidos, por un lado, a conseguir una mayor homogeneidad de dosis en los volúmenes tratados y, por otro, a disminuir las dosis administradas a OAR, fundamentalmente a pulmón y corazón. La mayor homogeneidad en la mama supone la eliminación de 
áreas de dosis fría y calientes y, potencialmente, unos mejores resultados estéticos y la disminución de dosis en OAR, una disminución de la toxicidad.

Un estudio randomizado, ha demostrado la posibilidad de reducir la toxicidad aguda con IMRT ${ }^{28}$, lo que abre la posibilidad a una disminución de toxicidad tardía y mejoría en aspectos cosméticos. Un estudio británico fase III que comparó 2D-RT con IMRT demostró una mejor apariencia de la mama a los 5 años aunque sin diferencias significativas en el análisis de calidad de vida ${ }^{29}$.

Diferentes estudios dosimétricos han demostrado una disminución de las dosis en pulmón y miocardio cuando se compara IMRT con técnicas de RT convencionales. Hurkmans comparó el riesgo cardiaco y pulmonar con un modelo de probabilidad de toxicidad en tejidos normales y encontró un menor riesgo de daño cardiaco tardío comparado con técnicas convenciona$\operatorname{les}^{30}$.
Existen dos formas diferentes de administrar IMRT en mama y sus ganglios regionales. A la primera de ellas podríamos denominar IMRT "convencional" que utiliza múltiples haces de tratamiento con diferentes ángulos y planificación inversa. La segunda (IMRT simple), se basa en los 2 haces tangenciales clásicos de la irradiación mamaria y puede realizarse con una planificación directa ("forward-planning") ${ }^{31}$. Esta técnica es más sencilla en su planificación y administración y acarrea un menor impacto en la carga de trabajo y tiempo de uso del acelerador. La primera permite una mayor conformación de dosis al volumen blanco por lo que se reducen significativamente los porcentajes de corazón y pulmón que reciben dosis altas pero, por otro lado, se aumenta el volumen de tejido sano que recibe una dosis baja ${ }^{32}$. La importancia del aumento de tejidos sanos que recibe una dosis baja y su repercusión en la toxicidad tardía y riesgo de segundos tumores está por determinar (Fig. 2).
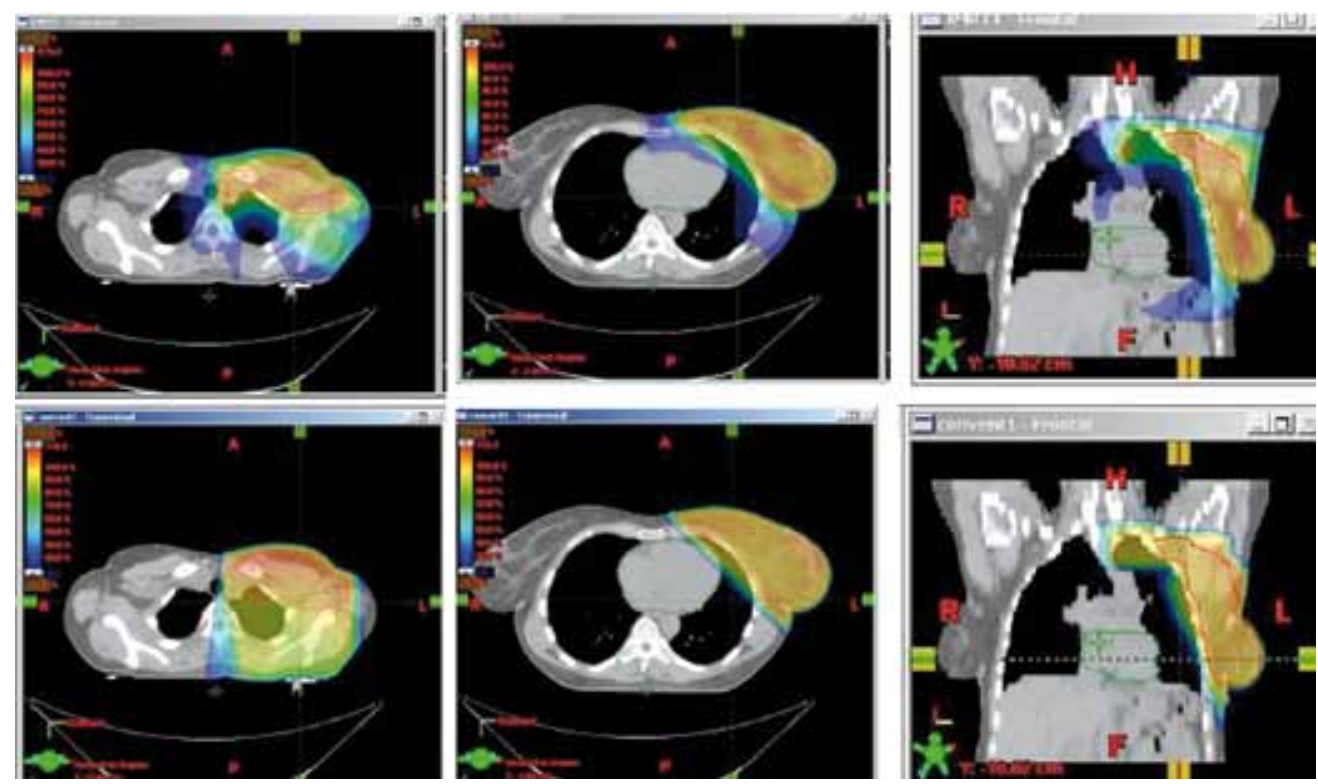

Figura 2. Dosimetrías comparativas: en la parte superior IMRT "convencional: las dosis altas (amarillonaranja) no salen fuera de la zona a tratar, pero las dosis bajas (azules) son más extensas que en la IMRT "simple" (parte inferior). 


\section{CONSIDERACIONES FINALES}

La IMRT supone un gran avance en los tratamientos radioterápicos por su capacidad de conformar la dosis de una forma muy ajustada al volumen blanco y por la creación de altos gradientes de dosis para proteger órganos de riesgo muy próximos. La apropiada aplicación clínica de esta tecnología requiere una cuidadosa consideración de las cuestiones relacionadas con la heterogeneidad de dosis y de las diferentes dosis-fracción resultantes, así como de la relevancia a largo plazo de las áreas tratadas con bajas dosis sin olvidar las incertidumbres relacionadas con el posicionamiento y la movilidad de los órganos a tratar. Es importante que las ventajas dosimétricas sean contrastadas en estudios prospectivos para poder llegar a un uso racional de una técnica más sofisticada y más exigente de los recursos sanitarios y conocer exactamente el beneficio clínico que obtenemos.

\section{BIBLIOGRAFÍA}

1. Galvin JM, Ezzell G, Eisbrauch A, Yu C, Butler $\mathrm{B}, \mathrm{XIAO} \mathrm{Y}$ et al. Implementing IMRT in clinical practice: A joint document of the American Society for Therapeutical Radiology and Oncology and the American Association of Physiscist in Medicine. Int J Rad Oncol Biol Phys 2004; 58: 1616-1634.

2. Pirzkall A, Carol M, Lohr F, Höss A, WannenMACHeR M, Debus J. Comparison of intensitymodulated radiotherapy with conventional conformal radiotherapy for comple-shaped tumors. Int J Rad Oncol Biol Phys 2000; 48: 1371-1380.

3. KuPPERSMith RB, GRECo SC and The BS. Intensity-modulated radiation therapy: first results with this new technology on neoplasm of the head and neck. Ear Nose Throat J 1999; 78: 238-241.

4. Vergeer MR, Doornaert PA, Rietveld DH, Leemans CR, Slotman BJ, LangendiJK JA. Intensitymodulated radiotherapy reduces radiationinduced morbidity and improves health-related quality of life: results of a nonrandomized prospective study using a standadized follow up program. Int J Rad Oncol Biol Phys 2009; 74: 1-8.
5. Chao KS, Majhall N, Huang CJ, Simpson J, PeREZ CA, HAugheY B et al. Intensity-modulated radiation therapy reduce late salivary toxicity without compromising tumor control in patients with orofaringeal carcinoma: A comparison with conventional techniques. Radioter Oncol 2001; 61: 275-280.

6. Eisbruch A, Harris J, Garden AS, Chao KS. Phase II multi-institutional study of IMRT for oropharyngeal cancer (RTOG 00-22): Early results. Int J Rad Oncol Biol Phys 2006; 66: S46.

7. Cheng JC, Chao KS, Low DA. Comparison of intensity modulated radiation therapy (IMRT) treatment techniques for nasopharyngeal carcinoma. Int J Cancer 2001; 96: 126-131.

8. Hunt MA, Zelefsky MJ, Wolden S, Chu CS, LoSAsso T, RosenzweIg $\mathrm{K}$ et al. Treatment planning and delivery of intensity modulated radiation therapy for primary nasopharynx cancer. Int J Rad Oncol Biol Phys 2001; 49: 623-632.

9. Kam MK, Leung SF, Zee B, Chor PHK, Chau MRC, CHeUng KY et al. Impact of intensity-modulated radiotherapy (IMRT) on salivary gland function in early-stage nasopharyngeal carcinoma (NPC) patients: A prospective randomized study. J Clin Oncol 2005; 23 (Suppl 1): 5501.

10. Pow EH, Kwong DL, McMillan AS, Wong MCM, Sham JST, Leung LHT et al. Xerostomia and quality of life after intensity-modulated radiotherapy vs. conventional radiotherapy for early stage nasopharyngeal carcinoma: Initial report on a randomized controlled clinical trial. Int J Rad Oncol Biol Phys 2006; 66: 981-991.

11. Bucci M, Xia P, Lee N, Fishbein N, Kramer A, WeinBERG $\mathrm{V}$ et al. Intensity-modulated radiation therapy for carcinoma of the nasopharynx: An update of the UCSF experience. J Rad Oncol Biol Phys 2004; 60: S317-S318.

12. Claus F, De Gersem De Wagter C, Van Severen $\mathrm{R}$, Vanhoutte I, Duthoy W et al. An implementation strategy for IMRT of ethmoid sinus cancer with bilateral sparing of the optic pathways. Int J Rad Oncol Biol Phys 2001; 51: 318-331.

13. Duthoy W, Boterberg T, Claus F, Ost P, Vakaet L, BRAI $S$ et al. Postoperative intensity-modulated radiotherapy in sinonasal carcinoma: Clinical results in 39 patients. Cancer 2005; 104: 71-82.

14. Kam MK, Chau RM, Suen J, Chol PH, Teo PM. Intensity-modulated radiotherapy in nasopharyngeal carcinoma: dosimetric advantage over conventional plans and feasibility of dose escalation. Int J Rad Oncol Biol Phys 2003; 56: 145-157. 
15. Wu Q, Mohan R, Morris M, Lauve A, Schmidt-UlRICH R. "Simultaneous integrated boost" IMRT of advanced head and neck squamous cell carcinoma. Int J Rad Oncol Biol Phys 2003; 56: 573-585.

16. Guerrero U, Clark C, Hansen V, Adams EJ, A`Herm R, Miles E et al. A phase I study of dose-escalated chemoradiation with accelerated intensity modulated radiotherapy in locally advanced head and neck cancer. Radiother Oncol 2007; 85: 36-41.

17. Chao KS, Ozygit G, Tran BNA, Cengiz M, DempSEY JF. Patterns of failure in patients receiving definitive and post-operative IMRT for head and neck cancer. Int J Rad Oncol Biol Phys 2003; 55: 312-321.

18. Dawson LA, Anzai Y, Marsh L, Martel MK, PaULINO A, SHIP JA et al. Patterns of local-regional recurrence following parotid-sparing conformal and segmental intensity-modulated radiotherapy for head and neck cancer. Int $\mathrm{J}$ Rad Oncol Biol Phys 2000; 46: 1117-1126.

19. Daisne JF, Sibomana M, Bol A, Doumont TH, LonNEUX M, GrÉGoIRE V. Evaluation of multimodality image (CT, MRI and PET) corregistration procedure on phantom and head and neck cancer patients. Radiother Oncol 2003; 69: 237-245.

20. ZelefsKy MJ, FuKs Z, HaPPERSETt L, LeE HJ, Ling CC, Burman ChM et al. Clinical experience with intensity-modulated radiation therapy (IMRT) in prostate cancer. Radiother Oncol 2000; 55: 241-249.

21. Zelefsky MJ, Fuks Z, Hunt M, Yamada Y, Marion $\mathrm{CH}$, Ling CC, et al. High-dose intensity-modulated radiation therapy for prostate cancer: early toxicity and biochemical outcome in 772 patients. Int J Rad Oncol Biol Phys 2002; 53: 1111-1116.

22. Zelefsky M, Chan H, Hunt M, Yamada Y, M-Shippy A, Amols H. Long-term outcome of high-dose intensity-modulated radiation therapy for patients with clinically localized prostate cancer. J Urol 2006; 176: 1415-1419.

23. Eade Tm, Hanlon AL, Horwitz EM, Buyyounouski MK, Hanks GE, Pollack A. What dose of external-beam radiation is enough for prostate cancer? Int J Rad Oncol Biol Phys 2007; 68: 682-689.

24. Cahlon O, Zelefsky MJ, Shippy A, Chan H, Fucks $\mathrm{Z}$, YAMADA $\mathrm{Y}$ et al. Intensity-modulated ra- diation therapy to a dose of 86.4 Gys for localized prostate cancer: Toxicity data nad preliminary biochemical outcomes. Int J Rad Oncol Biol Phys 2006; 66 (Supp 11 abs): S33.

25. Kupelian PA, Willoughby TR, Reddy CA. Hypofractionated intensity-modulated radioterapy (70 Gys at 2.5 Gy per fraction) for localized prostate cancer: Cleveland Clinic experience. Int J Rad Oncol Biol Phys 2007; 68: 1428-1430.

26. Lips I, Dehnad H, Kruger AB, van MoorselaAR J, van DER Heide U, Battermann $J$ et al. Healthrelated quality of life in patients with locally advanced prostate cancer after 76 Gys intensity-modulated radioterapy vs 70 Gys conformal radiotherapy in a prospective and longitudinal study. Int J Rad Oncol Biol Phys 2007; 69: 656-661.

27. NAMIKI SS, Ishidoya SS, Tochigi TT. Health-related quality of life after intensity-modulated radiation therapy for localized prostate cancer: comparison with conventional and conformal radiotherapy. Jap J Clin Oncol 2006; 36: 224-230.

28. Pignol JP, Olivotto I, Rakovitch E, Gardner S, Sixel K, Becкham W et al. A Multicenter randomized trial of breast intensity-modulated radiation therapy to reduce acute radiation dermatitis. Int J Rad Oncol Biol Phys 2008; 26: 2085-2092.

29. Donovan E, Bleakley N, Denholm E, Evans Ph, Gothard L, Hanson J et al. Randomised trial of standard 2D radiotherapy (RT) versus intensity modulated radiotherapy (IMRT) in patients prescribed radiotherapy. Radiother Oncol 2007; 82: 254-264.

30. Hurkmans CW, Cho BC, Damen E, ZiJp L, Mijnheer BJ. Reduction of cardiac and lung complication probabilities after breast irradiation using conformal radiotherapy with or without intensity modulation. Radioter Oncol 2002; 62: 163-171.

31. Vicini FA, Sharpe M, Kestin L, Martinez A, MitCHELl CK, WALLACE MF et al. Optimizing breast cancer treatment efficacy with intensity-modulated radiotherapy. Int J Rad Oncol Biol Phys 2002; 54: 1336-1344.

32. Beckham Wa, Popescu C, Patenaude V, Wai E, OLIVotTo IA. Is multibeam IMRT better than standard treatment for patients with left-sided breast cancer? Int J Rad Oncol Biol Phys 2007; 69: 918-924. 
